\title{
Evolutionary divergence and functions of the ADAM and ADAMTS gene families
}

Chad N. Brocker,' Vasilis Vasiliou' and Daniel W. Nebert ${ }^{2 *}$

'Molecular Toxicology and Environmental Health Sciences Program, Department of Pharmaceutical Sciences,

University of Colorado Denver, Aurora, CO 80045, USA

${ }^{2}$ Department of Environmental Health and Center for Environmental Genetics (CEG), University of Cincinnati Medical Center, Cincinnati, OH 45267-0056, USA

*Correspondence to: E-mail: dan.nebert@uc.edu; vasilis.vasilou@ucdenver.edu

Date received (in revised form): 28th May 2009

\begin{abstract}
The 'A-disintegrin and metalloproteinase' (ADAM) and 'A-disintegrin and metalloproteinase with thrombospondin motifs' (ADAMTS) genes make up two similar, yet distinct, gene families. The human and mouse genomes contain $2 \mathrm{I}$ and 24 putatively functional protein-coding ADAM genes, respectively, and 24 versus 32 putatively functional protein-coding ADAMTS genes, respectively. Analysis of evolutionary divergence shows that both families are unique. Each of the two families can be separated, if need be, into groups of more closely related members: six subfamilies for ADAM, four subfamilies for ADAMTS. The presence of both disintegrin and peptidase domains within the ADAM and ADAMTS proteins implies multiple biological roles within the cell. Membrane-anchored ADAM proteins are best known for their role in activating zymogens — including tumour necrosis factor- $\alpha$, epidermal growth factor (EGF) and amyloid precursor protein (APP). ADAM proteins can also participate in cell adhesion via their interaction with integrins in neighbouring cells. ADAMTS are secreted proteins that participate in extracellular matrix maintenance by way of their cleavage of procollagen and proteoglycans. ADAMTS proteins also are involved in coagulation by cleaving von Willibrand factor precursor protein. ADAM and ADAMTS proteins participate in a wide range of cellular processes, including cell adhesion and migration, ectodomain shedding, proteolysis, development, ovulation and angiogenesis. Because these enzymes are believed to play an important role in a number of pathologies, including Alzheimer's disease, rheumatoid arthritis, atherosclerosis, asthma and cancer progression, the products of the ADAM and ADAMTS genes represent promising drug targets for the prevention and management of a number of human diseases.
\end{abstract}

Keywords: ADAM, ADAMTS, gene family integrins, metalloproteinases, peptidase activity, procollagen, proteoglycans, epidermal growth factor domain, thrombospondins domain, potential drug targets, human genome

\section{Introduction}

The $A D A M$ and $A D A M T S$ gene families are members of the metalloproteinase superfamily, which comprises a diverse group of multi-domain transmembrane and secreted proteins with varied biological functions. To date, $21 A D A M$ (Table 1) and 24 ADAMTS (Table 2) protein-coding genes have been identified in the human genome, whereas 24 Adam and 32 Adamts protein-coding genes have been named in the mouse genome (http://www.genenames.org/). The human $A D A M$ gene family also has five confirmed pseudogenes namely, $A D A M 1, A D A M 3 A, A D A M 3 B, A D A M 5 P$ and $A D A M 21 P$ (although to be consistent with recommended nomenclature, the $A D A M 1, A D A M 3 A$ and $A D A M 3 B$ pseudogenes should end in the letter ' $P$ '). In addition, $A D A M 6$ is considered to be a likely $A D A M$ pseudogene. 
Table I. Human ADAM genes, as listed in the Human Gene Nomenclature Committee (HGNC) database

\begin{tabular}{|c|c|c|c|c|c|}
\hline $\begin{array}{l}\text { Gene } \\
\text { name }\end{array}$ & Other aliases & $\begin{array}{l}\text { Chromosomal } \\
\text { location }\end{array}$ & $\begin{array}{l}\text { RefSeq } \\
\text { RNA }\end{array}$ & $\begin{array}{l}\text { RefSeq } \\
\text { protein }\end{array}$ & $\begin{array}{l}\text { Amino } \\
\text { acid } \\
\text { number }\end{array}$ \\
\hline ADAM2 & $\begin{array}{l}\text { CTI5; FTNB; PH30; } \\
\text { CRYNI; CRYN2; PH-30b }\end{array}$ & $8 p \mid 1.2$ & NM00I 464.3 & NP00I 455.3 & 735 \\
\hline ADAM7 & EAPI; GP-83 & $8 p 21.2$ & NM0038I7.2 & NP003808.2 & 754 \\
\hline ADAM8 & MS2; CDI56; MGCI34985 & $10 q 26.3$ & NM00I I09.3 & NP00I I 00.2 & 856 \\
\hline ADAM9 & $\begin{array}{l}\text { MCMP; MDC9; Mltng; } \\
\text { KIAA002I }\end{array}$ & $8 p \mid 1.23$ & NM0038I 6.2 & NP003807.I & 819 \\
\hline ADAMIO & $\begin{array}{l}\text { kuz; ADI0; MADM; } \\
\text { CDI56c; HsTI87I7 }\end{array}$ & $15 q 2 ; 15 q 22$ & NM00III 0.2 & NP00IIOI.I & 748 \\
\hline ADAM I I & $M D C$ & $17 q 21.3$ & NM002390.4 & NP00238I.2 & 769 \\
\hline ADAMI2 & $\begin{array}{l}\text { MCMP; MLTN; MLTNA; } \\
\text { MCMPMltna }\end{array}$ & $10 q 26.3$ & NM003474.4 & NP003465.3 & 909 \\
\hline ADAMI5 & $M D C I 5$ & |q21.3 & NM0038I5.3 & NP003806.3 & 814 \\
\hline ADAMI 7 & $\begin{array}{l}\text { TACE; cSVP; CD/56b; } \\
\text { MGC7/942 }\end{array}$ & $2 p 25$ & NM003I83.4 & NP003I 74.3 & 824 \\
\hline ADAMI8 & $\begin{array}{l}\text { ADAM27; tMDCIII; } \\
\text { MGC4I836; MGC88272 }\end{array}$ & $8 p \mid 1.22$ & NM0 I4237.I & NP055052.I & 739 \\
\hline ADAMI9 & $\begin{array}{l}\text { MLTNB; FKSG34; } \\
\text { MADDAM }\end{array}$ & $5 q 32-q 33$ & NM023038.3 & NP075525.2 & 956 \\
\hline ADAM20 & & $14 q 24.1$ & NM0038I4.4 & NP003805.3 & 776 \\
\hline ADAM2I & ADAM3 I; MGCI 25389 & $14 q 24.1$ & NM0038I3.2 & NP003804.I & 722 \\
\hline ADAM22 & MDC2; MGCI49832 & $7 q 21$ & NM004I94.2 & NP004I85.I & 859 \\
\hline ADAM23 & $M D C 3$ & $2 q 33$ & NM0038I 2.2 & NP003803.I & 832 \\
\hline ADAM28 & $\begin{array}{l}\text { MDCL; ADAM23; } \\
\text { MDC-Lm; MDC-Ls; } \\
\text { eMDCII }\end{array}$ & $8 p 21.2$ & NMOI4265.4 & NP055080.2 & 775 \\
\hline ADAM29 & svph I & $4 q 34$ & NMOI 4269.4 & NP055084.3 & 820 \\
\hline ADAM30 & svph4 & $|p| 3-p||$ & NM021794.2 & NP068566.2 & 790 \\
\hline ADAM32 & $\begin{array}{l}\text { FLJ26299; FLJ29004; } \\
\text { ADAM32 }\end{array}$ & $8 p \mid 1.23$ & NMI 45004.5 & NP65944I.3 & 787 \\
\hline ADAM33 & $\begin{array}{l}\text { FLJ35308; FLJ3675 I; } \\
\text { MGC7I889; C20orfI 53; } \\
\text { DJ964F7.I; MGCI 49823; } \\
\text { DKFZP434K052I }\end{array}$ & $20 p / 3$ & NM025220.2 & NP079496.I & 813 \\
\hline ADAMDECI & MI2.219; FLJ79219 & $8 p 21.2$ & NM0I4479.3 & NP055294.I & 470 \\
\hline
\end{tabular}

ADAM pseudogenes include ADAMI, ADAM3A, ADAM3B, ADAM5P, ADAM6 and ADAM2IP; therefore, these are not included in the 'active' gene list, nor are they included in the dendogram in Figure 2. 
Table 2. Human ADAMTS genes, as listed in the HGNC database

\begin{tabular}{|c|c|c|c|c|c|}
\hline $\begin{array}{l}\text { Gene } \\
\text { name }\end{array}$ & Other aliases & $\begin{array}{l}\text { Chromosomal } \\
\text { location }\end{array}$ & RefSeq RNA & $\begin{array}{l}\text { RefSeq } \\
\text { protein }\end{array}$ & $\begin{array}{l}\text { Amino } \\
\text { acid } \\
\text { number }\end{array}$ \\
\hline ADAMTSI & C3-C5; METHI; KIAAI 346 & $2 \mid q 21.2$ & NM006988.3 & NP008919.3 & 967 \\
\hline ADAMTS2 & $\begin{array}{l}\text { NPI; PCINP; PCPNI; hPCPNI; } \\
\text { ADAM-TS2; ADAMTS-3 }\end{array}$ & 5qter & NM0I4244.2 & NP055059.2 & $|2| \mid$ \\
\hline ADAMTS3 & ADAMTS-4; KIAA0366 & $4 q 13.3$ & NM0 I4243.I & NP055058.I & 1205 \\
\hline ADAMTS4 & $\begin{array}{l}\text { ADMP-I; ADAMTS-2; ADAMTS-4; } \\
\text { KIAA0688 }\end{array}$ & |q21-q23 & NM005099.4 & NP005090.3 & 837 \\
\hline ADAMTS5 & ADMP-2; ADAMTSI I; FLJ36738 & $2 \mid q 21.3$ & NM007038.3 & NP008969.2 & 930 \\
\hline ADAMTS6 & ADAM-TS6 & $5 q 12$ & NMI9794I.2 & NP922932.2 & 1117 \\
\hline ADAMTS7 & ADAM-TS7; DKFZp434H2O4 & $15 q 24.2$ & NM0 I 4272.3 & NP055087.2 & 1686 \\
\hline ADAMTS8 & METH2; ADAM-TS8; FLJ4I 7 I 2 & $1 / q 25$ & NM007037.4 & NP008968.4 & 889 \\
\hline ADAMTS9 & FLJ42955; KIAAI3/2 & $3 p|4.3-p| 4.2$ & NMI82920.I & NP89I550.I & 1935 \\
\hline ADAMTSIO & WMS; ADAM-TSIO & $19 p \mid 3.3$ & NM030957.2 & NPI I 2219.2 & 1103 \\
\hline ADAMTSI 2 & PRO4389 & $5 q 35$ & NM030955.2 & NPII 2217.2 & 1594 \\
\hline ADAMTSI3 & $\begin{array}{l}\text { TTP; VWFCP; C9orf8; } \\
\text { VWF-CP; FLJ42993; } \\
\text { MGCI I 8899; MGCI I8900; } \\
\text { DKFZP434C2322 }\end{array}$ & $9 q 34$ & NMI39025.3 & NP620594.I & 1427 \\
\hline ADAMTSI 4 & FLJ32820 & $10 q 21$ & NMI39I55.2 & NP63I894.2 & 1226 \\
\hline ADAMTSI5 & MGCI 26403 & $1 \mathrm{lq} 25$ & NMI39055.I & NP620686.I & 950 \\
\hline ADAMTSI 6 & FLJI673I; ADAMTSI6s & $5 p \mid 5$ & NMI39056.2 & NP620687.2 & 1224 \\
\hline ADAMTSI 7 & FLJI6363; FLJ32769 & $15 q 24$ & NMI39057.2 & NP620688.2 & 1095 \\
\hline ADAMTSI8 & ADAMTS2I & $16 q 23$ & NMI99355.2 & NP955387.I & $122 \mid$ \\
\hline ADAMTSI9 & FLJI 6042 & $5 q 31$ & NMI 33638.3 & NP598377.3 & 1207 \\
\hline ADAMTS20 & GON-I & $12 q 12$ & NM025003.3 & NP079279.3 & 1910 \\
\hline ADAMTSLI & $\begin{array}{l}\text { C9orf94; PUNCTIN; ADAMTSRI; } \\
\text { FLJ35283; FLJ4I032; FLJ4689I; } \\
\text { MGC40I93; MGCI I 8803; } \\
\text { MGCI I 8805; } \\
\text { DKFZp686L03I30 }\end{array}$ & $9 p 22.2-p 22.1$ & NM00I040272.4 & NP00I 035362.3 & 1762 \\
\hline ADAMTSL2 & FLJ45 I 64; KIAA0605 & $9 q 34.2$ & NM00 I I 45320.1 & NP00 I I 38792.1 & 951 \\
\hline ADAMTSL3 & $\begin{array}{l}\text { KIAAI 233; MGCI507I6; } \\
\text { MGCI 507I7 }\end{array}$ & $15 q 25.2$ & NM2075I7.2 & NP997400.2 & 1691 \\
\hline ADAMTSL4 & TSRCI & |q2|.2 & NM0I 9032.4 & NP06I905.2 & 1074 \\
\hline ADAMTSL5 & THSD6 & $19 p \mid 3.3$ & NM213604.2 & NP998769.2 & 471 \\
\hline
\end{tabular}


Despite the presence of metallopeptidase (MP) domains, only 13 ADAM proteins exhibit peptidase activity. Five of the 24 ADAMTS genes lack MP domains and are proteolytically inactive. The remaining 19 appear to encode functional proteins with peptidase activity.

The two families encode proteins that are structurally similar and share a number of domains, including the prodomain (PD), reprolysin-like zinc-peptidase domain, disintegrin (DIS) domain and cysteine-rich region (CR). ADAM proteins also contain a $\mathrm{COOH}$-terminus transmembrane (TM) domain, which serves as a membrane anchor. Most ADAM proteins - the exceptions being ADAM10 and ADAM17 - also carry an epidermal growth factor (EGF)-like domain. ${ }^{1}$ ADAM proteins are key players in mediating ectodomain-shedding of several proteins, including tumour necrosis factor- $\alpha$ (TNF), transforming growth factor (TGF)- $\alpha$, EGF, amphiregulin (AREG) and Fas-ligand (FASLG).

'Ectodomain shedding' results in the cleavage of the ectopic portion of membrane-anchored proteins, which are released into the extracellular matrix (ECM). Ectodomain shedding is often followed by regulated intramembrane proteolysis (RIP), where the remaining intracellular portion is processed to form nuclear-targeted signals that regulate gene expression. Other ADAM substrates include cadherins, laminins and collagens in the ECM.

In addition to the PD, MP, DIS and cysteine-rich domains, proteolytic ADAMTS proteins include a thrombospondin (TS) type-I domain and are secreted into the ECM. ADAMTS proteins that are secreted into the ECM cleave type I, type II and type III procollagens. In addition, ADAMTS proteins are known to cleave large aggregating proteoglycans, known as aggrecans, which comprise a large component of cartilage, especially articular cartilage. ADAMTS proteins that are known to cleave aggrecans are referred to as aggrecanases. The ADAMTS gene family includes five ADAMTS-like (ADAMTSL) genes; these genes lack the PD, MP and DIS domains found in other ADAMTS proteins and are proteolytically inactive. ADAMTS dysregulation is associated with a number of connective tissue disorders, including Weill-Marchesani syndrome, Ehlers-Danlos syndrome type VIIC, some forms of encephalomyelitis, and arthritis.

The purpose of this review is threefold: first, to describe the domain structure of these two gene families; secondly, to review the known members of each family; and, lastly, to discuss briefly the functional relationships within each family subgroup.

\section{Domain structure of ADAM and ADAMTS proteins}

There are many structural and functional similarities between proteins encoded by the $A D A M$ and ADAMTS gene families. Both represent multidomain proteins, and the two families share several domains (Figure 1). There are many significant structural differences that account for their distinct biological functions, however. Both ADAM and ADAMTS proteins contain an $\mathrm{NH}_{2}$-terminus signalling sequence; the signalling sequence targets the protein to the secretory pathway. The PD (PF01562) follows the secretory signal found near the $\mathrm{NH}_{2}$-terminus. In ADAM proteins, the $\mathrm{PD}$ functions to inhibit catalytic activity, until activation via a cysteine-switch mechanism occurs. The PD regulates enzyme activity by blocking the peptidase active-site. Cleavage of the PD is believed to mediate ADAM catalytic activity. Many ADAM proteins contain a furin-recognition motif (RXXR

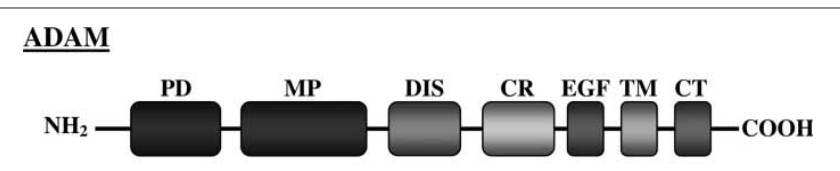

\section{$\underline{\text { ADAMTS }}$}

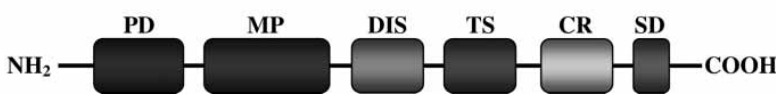

Figure I. The individual modular structures of the ADAM and ADAMTS proteins. CR, cysteine-rich domain; CT, cytoplasmic tail; DIS, disintegrin domain; EGF, epidermal growth factor-like domain; MP, metallopeptidase domain; PD, prodomain; SD, spacer domain; TM, transmembrane domain;

TS, thrombospondin-like domain. 
sequence) located immediately following the PD and prior to the MP domain. Furins belong to a family of proprotein convertases which cleave latent precursor proteins at paired basic amino acid processing sites; the cleavage event produces the mature/active protein.

Mutation studies with the $A D A M 10$ gene found that altering the furin-recognition motif results in an inactive enzyme. ${ }^{2}$ The PD is processed and removed within the trans-Golgi network, prior to either transport to the cell membrane or secretion. ${ }^{3}$ Proteolytic ADAMTS also contain at least one furin-like recognition motif, located between the PD and MP domains, which may mediate enzyme activity. Two ADAM proteins, ADAM8 and ADAM28, are activated via an autocatalytic mechanism. $^{4,5}$

ADAM and ADAMTS enzymes are classified as zinc endopeptidases and contain a conserved zincbinding motif within the reprolysin family zinc metallopeptidase domain (PF01421). The amino acid sequence making up the zinc-binding motif, 'HEXGHXXGXXHZ' and a downstream 'Met Turn' are conserved throughout the ADAMTS family members (the exception being the five ADAMTSL genes). Several ADAM family members (including ADAM2, ADAM11, ADAM18, ADAM23 and ADAM32) lack all three conserved histidines found within the zinc-binding motif; absence of these residues prevents zinc binding and subsequent activity. ADAM22 lacks two of the three conserved histidines, which also prevents peptidase activity. ${ }^{6}$ The inability to act as a peptidase suggests that these regions might facilitate protein folding or possibly participate in proteinprotein interactions.

The MP domain (Figure 1) is followed immediately by the DIS domain (PF00200). Disintegrins were originally identified as small proteins found in snake venom that block platelet aggregation via their interaction with integrins. ${ }^{7}$ Integrins are found on the cell surface, where they mediate important cell-cell and cell-ECM interactions. A wide variety of integrins are located on the cell surface; these can bind to the DIS domains found within ADAM proteins and participate in cell adhesion. ${ }^{8}$ A stretch of 14 amino acids within the DIS domain, referred to as the 'disintegrin loop', facilitates integrin binding. 9 "The DIS domain found in ADAMTS proteins lacks a number of conserved cysteine residues found within the snake venom disintegrins.

It is believed that the DIS domain in ADAMTS proteins may play a role in enzyme activity and substrate specificity. ${ }^{10}$ The ability of the ADAMTS DIS domain directly to bind integrins has not yet been identified.

The DIS domain in ADAM proteins is followed by a CR or cysteine-rich domain (PF08516). In ADAM13, the CR was found to regulate peptidase activity. $^{11}$ The crystal structure of ADAMTS revealed that the $\mathrm{CR}$ stacks against the peptidase active site and may therefore regulate enzyme activity. $^{12}$

The carboxy $(\mathrm{COOH})$ region of ADAM proteins (Figure 1) contains an EGF-like domain (PF07974), a TM domain and a cytoplasmic tail (CT) region. The EGF domain is found in all ADAMS proteins (except ADAM10 and ADAM17) and is immediately downstream of the CR. ${ }^{1}$ The exact function of the EGF domain has yet to be determined. The TM domain is responsible for anchoring the protein to the cell membrane. Following the TM domain is the CT, which participates in intracellular signalling. ${ }^{13}$ The CT domain varies greatly among ADAM family members.

ADAMTS proteins lack the EGF, TM and CT domains found in the ADAM proteins. Instead, the ADAMTS proteins contain a TS domain (PF00090), found between the DIS domain and the CR domain (Figure 1); also, following the CR domain, ADAMTS proteins have a spacer domain (SD) (PF05986). The COOH-terminal TS domain found in ADAMTS proteins participates in cellcell interactions, angiogenesis and apoptosis. ${ }^{14,15}$

The number of TS domains varies among the ADAMTS proteins. The long isoforms of ADAMTS9 and ADAMTS20 both contain 15 TS repeats. ${ }^{16,17}$ ADAMTS4, by contrast, contains only a single TS domain. ${ }^{18}$ The most $\mathrm{COOH}$-terminal ADAMTS domain is the SD. The ADAMTS SD 
was shown to mediate substrate-binding affinity and proteolysis. ${ }^{19}$ The structural elements in these proteins (Figure 1) confer a wide range of biological functions.

\section{The human ADAM gene family}

ADAM proteins were first identified as sperm-associated enzymes. ${ }^{20}$ The understanding and importance of this gene family is increasing dramatically. Despite sharing a number of domains, the 21 genes and six pseudogenes that make up the human $A D A M$ gene family exhibit a diverse range of structural and functional features. The 21 protein-coding genes can be divided into six subfamilies, designated $A$ to $F$, based on protein sequence similarity, as indicated in the human ADAM dendrogram (Figure 2, top). Only 13 of these 21 genes encode proteins with protease activity. $A D A M 12, \quad A D A M 15, \quad A D A M 19$, ADAM22, ADAM28, ADAM29, ADAM33 and $A D A M D E C 1$ express multiple variants, which further diversifies the gene family. The ADAM proteins contribute to a wide array of biological processes, including cell adhesion, migration and signalling. Furthermore, these genes are thought to play pivotal roles in a number of pathologies, including cancer, arthritis, asthma, cardiovascular disease and Alzheimer's disease, to name but a few.

\section{ADAM subfamily $A$}

$A D A M 11, A D A M 22$ and $A D A M 23$ make up ADAM subfamily $A$ (Figure 2, top). All three proteins lack critical residues found within the peptidase domain and are considered proteolytically inactive. ADAM22 transcribes at least five variants, which encode five different protein isoforms; little is known about the precise function of ADAM22 and its variants. The function of the remaining two genes in this subfamily also remains relatively unexplored. Interestingly, the expression of all three ADAM proteins is predominantly confined to the central and peripheral nervous systems, hence, they are believed to play an important role in neuronal function and development. ${ }^{21,22}$ Studies have indicated non-redundant roles for ADAM11, ADAM22 and ADAM23 in the brain, based on the phenotypes displayed in knockout mice. Mice lacking functional Adam11 exhibit decreased pain sensation and display learning deficiencies. ${ }^{23}$ Adam22(-/-) knockout mice exhibit hypermyelination of peripheral nerves and die prior to weaning. ${ }^{24}$ Disruption of the mouse Adam 23 gene results in ataxia and premature death. ${ }^{25}$

\section{ADAM subfamily $B$}

This group contains four genes: $A D A M 2$, $A D A M 18, A D A M 32$ and $A D A M 9$ (Figure 2, top). The first three of these genes in this subfamily lack peptidase activity and are associated with sperm development and fertilisation. Adam 2 in mice is involved in spermatogenesis and fertility, but the exact role in humans has yet to be determined. Little is known about the function of ADAM18; however, it is expressed in human sperm, indicating that it may also be involved in spermatogenesis. ${ }^{26}$ ADAM32 is predominantly expressed in the testis and may also participate in sperm development or possibly fertilisation. ${ }^{27} A D A M 9$ is the only gene in this subfamily that contains potential $\mathrm{SH} 3$ binding sites in its CT. It is also the only member that has $\alpha$-secretase activity and can metabolise Alzheimer's amyloid precursor protein (AAP) towards the nonamyloidogenic pathway. This potentially protects the brain from plaque formation during Alzheimer's disease. Polymorphisms within the ADAM9 promoter region are associated with protection against sporadic Alzheimer's disease. ${ }^{28}$

\section{ADAM subfamily $C$}

Subgroup C contains four genes (Figure 2, top), including $A D A M 20, A D A M 21, A D A M 29$ and $A D A M 30$. All of the enzymes exhibit protease activity, with the exception of ADAM29. ADAM29 encodes four splice variants that differ in the composition of the $5^{\prime}$-untranslated region (UTR); nothing is known about the function of ADAM29 isoforms. ADAM29 is highly expressed in human testis and is believed to play a role during 
spermatogenesis. $^{29}$ ADAM20 and ADAM21 share 50 per cent protein identity. Both enzymes are predominantly expressed in the testis and possibly function in sperm-egg cell fusion, based on homology to mouse Adam $1 .{ }^{30}$ In rodents, Adam 21 was reported to facilitate neurogenesis and neuronal plasticity. ${ }^{31}$ ADAM30 is also highly expressed in the testis. $^{32}$

\section{ADAM subfamily $D$}

ADAM subfamily $D$ contains $A D A M 7, A D A M 28$ and $A D A M D E C 1$ (Figure 2, top). The three genes make up a cluster on human chromosome $8 \mathrm{p} 21$ and are believed to be the result of gene duplication events. ${ }^{33}$ The genes also share a high degree of nucleotide and of amino acid homology. ADAM7 protein does not contain the conserved peptidase motif required for catalytic activity. By contrast, ADAM28 and ADAMDEC1 are considered to be functional proteases. ADAMDEC1, or decysin, is unique among ADAM members, in that it completely lacks a CR domain and contains only a portion of the DIS domain. ${ }^{33}$ The gene is therefore referred to as being 'ADAM-like' and structurally distinct from other ADAM family members. The loss of downstream exons in ADAMDEC1 may be the result of an incomplete gene duplication process. ADAMDEC1 encodes three transcripts; however, two of the three transcripts are candidates for nonsense-mediated decay and might not be protein-coding.

The $A D A M 28$ gene transcribes two variants. The longer transcript encodes a 775-amino acid protein. The shorter variant contains an alternative $3^{\prime}$ coding region and $3^{\prime}-\mathrm{UTR}$, resulting in a much shorter protein. The shorter $\mathrm{COOH}$-terminus lacks both the TM and cytosolic domains and, similar to ADAMTS proteins, is secreted into the ECM. ADAM28 is highly expressed in the epididymis and, like many other $A D A M$ genes, is believed to participate in sperm development. ${ }^{4}$ Recently, ADAM28 was found to mediate epithelialmesenchymal signalling during mammalian tooth development. ${ }^{34}$ ADAM7 has also been associated with sperm development. ${ }^{35}$ The function of
ADAMDEC1 is not completely understood; however, expression was identified in activated mouse and human dendritic cells, indicating that it might participate in modulating the immune response. $^{36}$

\section{ADAM subfamily $E$}

Subfamily $E$ includes $A D A M 8, A D A M 12$, $A D A M 15, A D A M 19$ and $A D A M 33$ (Figure 2, top). All of these genes code for ADAM proteins that are catalytically active. $A D A M 12, A D A M 19$ and $A D A M 33$ each encode two variants, and ADAM15 encodes six. The ADAM12 and ADAM19 variants differ in the composition of their COOH-termini. Similar to ADAM28, the longer ADAM12 transcript is the classical membrane-anchored form of the protein. The shorter transcript lacks a TM domain and is secreted into the ECM. It is unclear if a similar situation exists between the ADAM19 variants. The second ADAM33 splice variant lacks an internal exon and encodes a shorter protein. $A D A M 15$ also encodes a number of variant transcripts. The exact function of each variant has yet to be determined, but the isoforms have been shown to have differential effects on cell adhesion and migration. ${ }^{37}$

There are a number of proline-rich motifs (PXXP) located within the CT region of ADAM12, ADAM15, ADAM19 and ADAM33. ${ }^{13}$ PXXP motifs are known to bind to SH3 domains, indicating intracellular protein-protein interactions. Interestingly, ADAM15 variants differ in the number of PXXP motifs found in the CT; this suggests that the variants may interact with different cytosolic proteins. A positive association has been found between asthma and $A D A M 33$ polymorphisms in several populations; however, the exact role of these polymorphisms remains unknown. ${ }^{38,39}$

ADAM8 shares the least homology (Figure 2, top) to the other subfamily $E$ members and encodes a single transcript. Studies of asthma in mice showed a strong association between the Adam8 protein, also called CD185, and disease progression. ${ }^{40}$ ADAM8 is predominantly expressed 


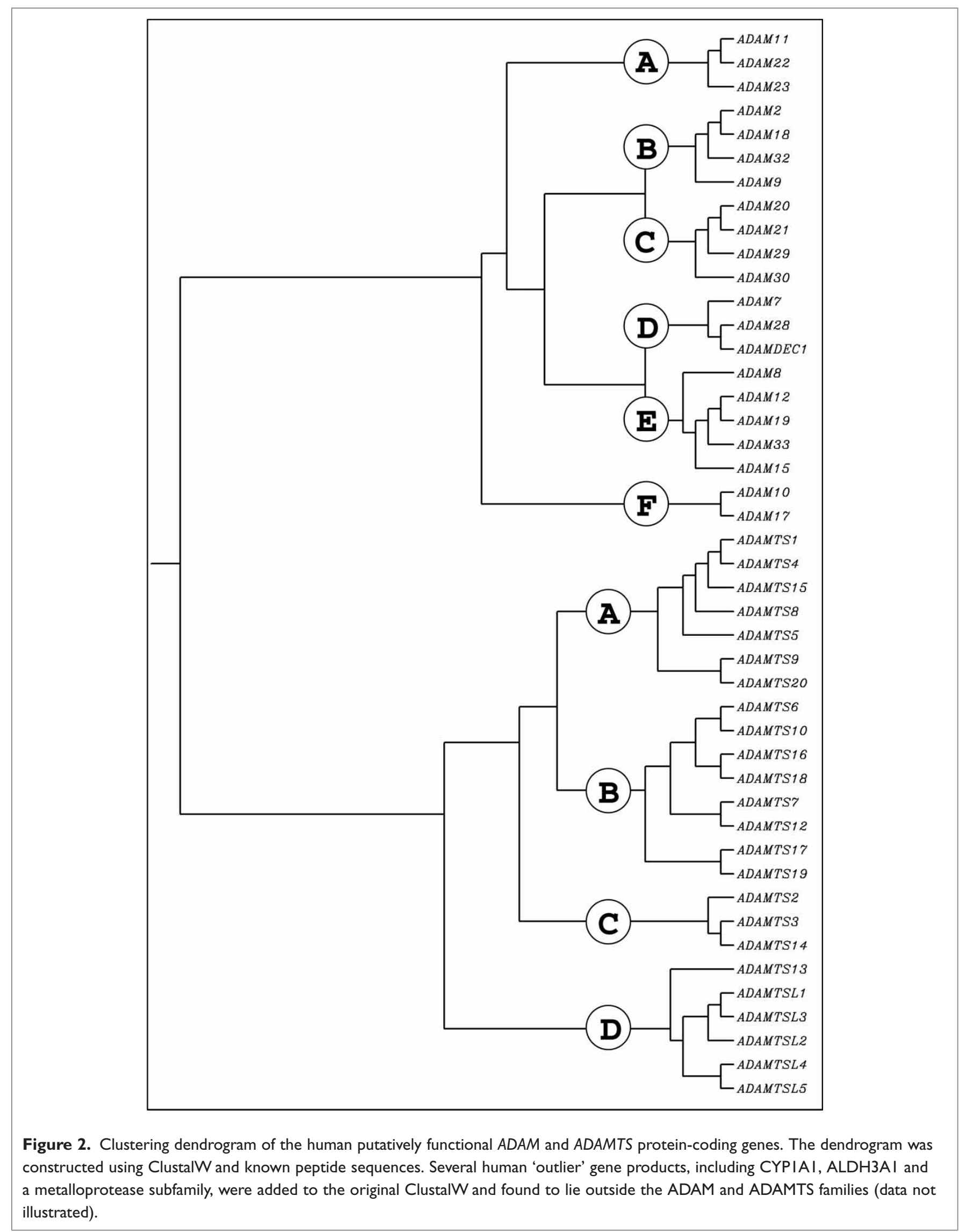


in haematopoietic cells and is upregulated during inflammation, which may explain the role it plays in asthma. ${ }^{41}$

\section{ADAM subfamily $\boldsymbol{F}$}

The final $A D A M$ gene subfamily contains only two genes, $A D A M 10$ and $A D A M 17$. As depicted in the human ADAM dendrogram (Figure 2, top), these two proteins are distinctly more evolutionarily separated from the rest of the $A D A M$ gene family. ADAM10 and ADAM17 are the only ADAM proteins that do not contain EGF-like domains. Both proteins bind zinc and are catalytically active against a number of protein substrates. The two proteins also contain potential SH3-binding sites located within the CT region. ${ }^{13}$ ADAM10 and ADAM17 are both considered as sheddases (also called secretases) and cleave the extracellular domain of membrane-bound proteins, resulting in the release of cytokines, growth factors, adhesion molecules and other enzymes.

To date, $A D A M 17$ is among the best-studied genes in the ADAM family. Also known as TNF convertase, ADAM17 cleaves TNF to its active form, which then modulates immune and pro-inflammatory responses. In addition to TNF, ADAM17 is the major sheddase for epiregulin, amphiregulin and heparin-binding EGF-like growth factors. ${ }^{42}$ Recently, the oxidative stress-induced oxidation of ADAM17 was found to enhance peptidase activity; redox sensitivity was attributed to thiol-disulphide bonds formed between the DIS and CR domains found in the extracellular domain, suggesting that many ADAM and ADAMTS proteins might be redox regulated. ${ }^{43}$ ADAM17 also cleaves membrane-tethered sonic hedgehog (ShhNp), possibly implicating ADAM proteins in the control of cell growth and patterning during development. ${ }^{44}$ In addition, ADAM17 is upregulated in inflammatory bowel diseases. ${ }^{45}$

ADAM proteins are linked to gene regulation by way of regulated RIP, in which proteolytic cleavage of a transmembrane protein produces signals that are subsequently targeted to the nucleus. An example is the cleavage of Notch by ADAM10. A number of ADAM proteins, including ADAM10 and ADAM17, are upregulated during tumour cell invasion into surrounding tissues. ${ }^{46}$ ADAM10 is the major protease responsible for the release of EGF and betacellulin. ADAM10 was also found to contribute to TNF shedding in cells that lack ADAM17, indicating a functional overlap between the two genes, despite sharing only 25 per cent amino acid identity. ${ }^{47}$ Both ADAM10 and ADAM17 also cleave AAP. This cleavage of AAP prevents the formation of the pathogenic amyloid- $\beta$ fragment responsible for Alzheimer's disease; many studies have therefore begun to focus on this therapeutic potential.

\section{The human ADAMTS gene family}

The 24 members of the ADAMTS gene family can be divided into four major subfamilies, based on peptide sequence similarity, as indicated in the ADAMTS dendrogram (Figure 2, bottom). The gene family as a whole has alternatively been referred to as the 'adamalysin-thrombospondins'. ${ }^{48}$ Originally, there was some overlap in ADAMTS gene nomenclature, and many of these enzymes had different names. ADAMTS5 and ADAMTS11 were both used to describe aggrecanase-2; subsequently, ADAMTS5 became the official name for the protein. ADAMTS13 was originally called von Willebrand factor cleaving protease. Pro-collagen I $\mathrm{N}$-proteinase is now referred to as ADAMTS2. Furthermore, five ADAMTSL genes have recently been identified. These members lack the PD, MP and DIS domains found in other ADAMTS proteins; however, the remaining domain organisation is remarkably similar.

\section{ADAMTS subfamily $A$}

The seven members of subfamily $A$ include ADAMTS1, ADAMTS4, ADAMTS5, ADAMTS8, ADAMTS9, ADAMTS15 and ADAMTS20 (Figure 2, bottom). Members within this subgroup contain the widest range of TS repeats. ADAMTS4 contains only a single TS domain, whereas ADAMTS9 and ADAMTS20 contain 15 repeats. ${ }^{48}$ 
ADAMTS9 and ADAMTS20 contain a gonadal (GON) domain (PF08685), indicating a possible role in gonadal development. ${ }^{49}$ The proteins in this subgroup all cleave the major cartilage proteoglycan, aggrecan, and are often referred to as aggrecanases. ADAMTS1, ADAMTS4 and ADAMTS9 also cleave the related proteoglycan, versican. ${ }^{50}$ Due to the importance of aggrecan and versican in cartilaginous tissue, many of these ADAMTS proteins have been implicated in the pathogenesis of arthritic disease. ${ }^{51,52}$

\section{ADAMTS subfamily $B$}

ADAMTS subclass $B$ (Figure 2, bottom) is the largest subclass and contains ADAMTS6, ADAMTS7, ADAMTS10, ADAMTS12, ADAMTS16, ADAMTS17, ADAMTS18 and ADAMTS19. The eight members in this group contain between five and eight TS repeats. ${ }^{48}$ ADAMTS7 and ADAMTS12 also contain a mucin domain (PF01456), located within the cluster of TS repeats in the $\mathrm{COOH}$-terminus. The domain is predicted to be heavily O-glycosylated, suggesting that the proteins may act as proteoglycans within certain tissues. ${ }^{53}$ ADAMTS10, ADAMTS12, ADAMTS17 and ADAMTS19 contain an additional protease-andlacunin (PLAC) domain (PF08686). ${ }^{48}$ Other ECM proteins containing this domain are involved in tissue development and remodelling. ${ }^{54}$

There is little information available related to the function of ADAMTS6. Both ADAMTS7 and ADAMTS12 cleave cartilage oligomeric matrix protein (COMP) and are upregulated in patients with rheumatoid arthritis, indicating that the genes encoding these proteins may play a significant part in the pathogenic progression of this disease. ${ }^{50}$ Mutations in the ADAMTS10 gene are responsible for the autosomal recessive form of WeillMarchesani syndrome, which is a rare connective tissue disorder. ${ }^{49}$ The main physiological substrate for ADAMTS10 still remains unknown. ADAMTS16 was recently found to cleave $\alpha_{2}$-macroglobulin (A2M). In addition, A2M is expressed in parietal granulosa cells during follicular development. $^{56}$ The functions and preferred substrates for ADAMTS17, ADAMTS18 and ADAM19TS remain unknown.

\section{ADAMTS subfamily C}

Subfamily C contains ADAMTS2, ADAMTS3 and ADAMTS14 (Figure 2, bottom). ADAMTS2 and ADAMTS14 both encode two transcript variants. The shorter ADAMTS2 variant lacks a number of TS repeats found in the $\mathrm{COOH}$-terminal region of the protein. The ADAMTS14 variant lacks a small region of coding sequence, resulting in the exclusion of three amino acids. All three members of this subgroup contain a PLAC domain within the $\mathrm{COOH}$-terminus, similar to those found in subfamily B. Furthermore, each of the ADAMTS2, ADAMTS3 and ADAMTS14 proteins contains a total of four TS repeats. ${ }^{48}$ All members of this group are involved in the $\mathrm{NH}_{2}$-terminal cleavage of procollagen to collagen and are also referred to as procollagen $\mathrm{N}$-proteinases. Mutations in the ADAMTS2 gene cause Ehlers-Danlos syndrome type VIIC, which is characterised by extreme skin fragility. Moreover, Adamts3 and Adamts14 are unable to compensate for Adamts 2 deficiency in Adamts2(-/-) knockout mice, indicating that the proteins may have distinct biological roles in ECM formation and maintenance. ${ }^{58}$

\section{ADAMTS subfamily $D$}

The genes that comprise subfamily $D$ contain significant structural differences, when compared with genes in the other ADAMTS subfamilies. This subfamily contains ADAMTS13 (Figure 2, bottom), as well as the five ADAMTSL genes. The ADAMTS13 gene contains 29 exons and encodes at least three transcriptional variants. One transcript lacks an internal coding region that results in the removal of 56 amino acids from the COOH-terminus, when compared with the longest form. Another variant lacks an additional upstream coding segment that codes for 31 amino acids. The PD of ADAMTS13 is significantly truncated, compared with the other ADAMTS subfamily genes. Moreover, ADAMTS13 is the only gene 
in the ADAMTS gene family to contain a CUB domain (PF00431). ${ }^{58}$ The CUB (Complement subcomponents C1r/C1s, (sea urchin epidermal growth factor1 [UEGF]) and bone morphogenetic protein-1 [BMP1])) domain is an extracellular motif consisting of $\sim 110$ residues which is found in a wide range of developmentally regulated proteins. ${ }^{59}$ ADAMTS13 was originally identified as the protease responsible for cleaving the von Willebrand factor, which is required for normal blood clotting. Mutations in the ADAMTS13 gene are the cause of hereditary thrombotic thrombocytopenic purpura (TTP). Moreover, sporadic TTP is an autoimmune disease resulting from ADAMTS13 inhibition by antibodies.

The bulk of subfamily $D$ is made up of the five proteolytically inactive ADAMTSL genes (Figure 2, bottom). ADAMTSL proteins lack many $\mathrm{NH}_{2}$-terminal features, including the PD, MP and DIS domains. They contain the characteristic TS repeats, as well as the SD and CR domains. Most ADAMTSL proteins also feature a PLAC domain, with the exception of ADAMTSL5. Interestingly, both ADAMTSL1 and ADAMTSL3 are predicted to contain immunoglobulin-like (PF00047) and immunoglobulin I-set domains (PF07679), which are found in a number of cell adhesion molecules. ADAMTSL1 can encode either 1,762 or 525 residue proteins; the shorter isoform utilises an alternative $3^{\prime}$ exon containing a premature stop codon, which results in subsequent $\mathrm{COOH}-$ terminus truncation.

ADAMTSL1 is N-glycosylated and secreted into the ECM. ${ }^{60}$ ADAMTSL2 also encodes a secreted glycoprotein. ${ }^{61}$ Two ADAMTSL2 transcripts have been identified, which vary in their $5^{\prime}$-UTR sequences. Mutations in the ADAMTSL2 gene result in dysregulation of TGF- $\beta$ signalling and are associated with geleophysic dysplasia (happy facial expression, short stature and limb abnormalities).

Little is known about the function of ADAMTSL3. Like the previously mentioned ADAMTSL proteins, ADAMTSL3 is a secreted glycoprotein; it may play a role in mediating cellcell interactions or in the assembly of extracellular matrices. $^{62}$ ADAMTSL3 mutations are also commonly associated with colorectal cancers. ${ }^{63}$ The ADAMTSL4 gene is closely linked to $A D A M 15$ and may have common ancestry; ADAMTSL4 is believed to originate from the interrupted inversion of a common familial ADAMTS gene. ${ }^{64}$ Mutations in the ADAMTSL4 gene were recently found to cause ectopia lentis, an autosomal recessive disorder characterised by lens displacement. $^{65}$ The function of ADAMTSL5 has yet to be determined.

\section{Conclusions}

The ADAM and ADAMTS genes comprise two important metalloproteinase subgroups. Both the ADAM and the ADAMTS gene families contain very similar domain organisation; however, the differences confer distinct biological functions. The ADAM family proteins are membrane-anchored and facilitate ectodermal shedding of growth factors and cell-signalling peptides. The proteins also participate in cell adhesion via their interaction with integrins. ADAMTS proteins lack the TM domain required for membrane association and are instead secreted extracellularly, where they cleave a diverse range of substrates. These two gene families participate in a wide range of physiological processes, including developmental regulation, inflammation, cell adhesion, cell signalling and angiogenesis. The biological significance of these proteins is emphasised by their strong association with many common pathological conditions, including asthma, arthritis and cancer. Our understanding of these proteins has grown immensely over the past decade, but the physiological and pathophysiological functions of many $A D A M$ and $A D A M T S$ genes have yet to be determined. Future investigations will no doubt highlight their potential as important therapeutic targets.

\section{Acknowledgments}

We thank our colleagues for valuable discussions and careful reading of this manuscript. The writing of this article was supported, in part, by NIH grants R01 EY11490 (V.V.) and P30 ES06096 (D.W.N.). 


\section{References}

1. Janes, P.W., Saha, N., Barton, W.A., Kolev, M.V. et al. (2005), 'Adam meets Eph: An ADAM substrate recognition module acts as a molecular switch for ephrin cleavage in trans', Cell Vol. 123, pp. 291-304.

2. Anders, A., Gilbert, S., Garten, W., Postina, R. et al. (2001), 'Regulation of the alpha-secretase ADAM10 by its prodomain and proprotein convertases', FASEB J. Vol. 15, pp. 1837-1839.

3. Lum, L., Reid, M.S. and Blobel, C.P. (1998), 'Intracellular maturation of the mouse metalloprotease disintegrin MDC15', J.Biol. Chem. Vol. 273, pp. 26236-26247.

4. Howard, L., Maciewicz, R.A. and Blobel, C.P. (2000), 'Cloning and characterization of ADAM28: Evidence for autocatalytic pro-domain removal and for cell surface localization of mature ADAM28', Biochem. J. Vol. 348, pp. 21-27.

5. Schlomann, U., Wildeboer, D., Webster, A., Antropova, O. et al. (2002), 'The metalloprotease disintegrin ADAM8. Processing by autocatalysis is required for proteolytic activity and cell adhesion', J. Biol. Chem. Vol. 277, pp. 48210-48219.

6. Andreini, C., Banci, L., Bertini, I., Elmi, S. et al. (2005), 'Comparative analysis of the ADAM and ADAMTS families', J. Proteome Res. Vol. 4, pp. $881-888$.

7. Niewiarowski, S., McLane, M.A., Kloczewiak, M. and Stewart, G.J. (1994), 'Disintegrins and other naturally occurring antagonists of platelet fibrinogen receptors', Semin. Hematol. Vol. 31, pp. 289-300.

8. Tomczuk, M., Takahashi, Y., Huang, J., Murase, S. et al. (2003), 'Role of multiple beta1 integrins in cell adhesion to the disintegrin domains of ADAMs 2 and 3', Exp. Cell Res. Vol. 290, pp. 68-81.

9. White, J.M. (2003), 'ADAMs: Modulators of cell-cell and cell-matrix interactions', Curr. Opin. Cell Biol. Vol. 15, pp. 598-606.

10. De Groot, R., Bardhan, A., Ramroop, N., Lane, D.A. et al. (2009), 'Essential role of the disintegrin-like domain in ADAMTS13 function', Blood Vol. 113, pp. 5609-5616.

11. Smith, K.M., Gaultier, A., Cousin, H., Alfandari, D. et al. (2002), 'The cysteine-rich domain regulates ADAM protease function in vivo', J. Cell Biol. Vol. 159, pp. 893-902.

12. Gerhardt, S., Hassall, G., Hawtin, P., McCall, E. et al. (2007), 'Crystal structures of human ADAMTS-1 reveal a conserved catalytic domain and a disintegrin-like domain with a fold homologous to cysteine-rich domains', J. Mol. Biol. Vol. 373, pp. 891-902.

13. Edwards, D.R., Handsley, M.M. and Pennington, C.J. (2008), 'The ADAM metalloproteinases', Mol. Aspects Med. Vol. 29, pp. 258-289.

14. Iruela-Arispe, M.L., Lombardo, M., Krutzsch, H.C., Lawler, J. et al. (1999), 'Inhibition of angiogenesis by thrombospondin-1 is mediated by 2 independent regions within the type 1 repeats', Circulation Vol. 100, pp. $1423-1431$.

15. Guo, N., Krutzsch, H.C., Inman, J.K. and Roberts, D.D. (1997), 'Thrombospondin 1 and type I repeat peptides of thrombospondin 1 specifically induce apoptosis of endothelial cells', Cancer Res. Vol. 57, pp. $1735-1742$.

16. Somerville, R.P., Longpre, J.M., Jungers, K.A., Engle, J.M. et al. (2003), 'Characterization of ADAMTS-9 and ADAMTS-20 as a distinct ADAMTS subfamily related to Caenorhabditis elegans GON-1', J. Biol. Chem. Vol. 278, pp. 9503-9513.

17. Llamazare, M., Cal, S., Quesada, V. and Lopez-Otin, C. (2003), 'Identification and characterization of ADAMTS-20 defines a novel subfamily of metalloproteinases-disintegrins with multiple thrombospondin-1 repeats and a unique GON domain', J. Biol. Chem. Vol. 278, pp. 13382-13389.

18. Tortorella, M.D., Burn, T.C., Pratta, M.A., Abbaszade, I. et al. (1999), 'Purification and cloning of aggrecanase-1: A member of the ADAMTS family of proteins', Science Vol. 284, pp. 1664-1666.

19. De Groot, R., Bardhan, A., Ramroop, N., Lane, D.A. et al. (2009), 'Essential role of the disintegrin-like domain in ADAMTS13 function', Blood Vol.

20. Blobel, C.P. (1997), 'Metalloprotease-disintegrins: Links to cell adhesion and cleavage of TNF alpha and Notch', Cell Vol. 90, pp. 589-592.
21. Sagane, K., Ohya, Y., Hasegawa, Y. and Tanaka, I. (1998), 'Metalloproteinase-like, disintegrin-like, cysteine-rich proteins MDC2 and MDC3: Novel human cellular disintegrins highly expressed in the brain', Biochem. J. Vol. 334, pp. 93-98.

22. Sagane, K., Yamazaki, K., Mizui, Y. and Tanaka, I. (1999), 'Cloning and chromosomal mapping of mouse ADAM11, ADAM22 and ADAM23', Gene Vol. 236, pp. 79-86.

23. Takahashi, E., Sagane, K., Oki, T., Yamazaki, K. et al. (2006), 'Deficits in spatial learning and motor coordination in ADAM11-deficient mice', BMC Neurosci. Vol. 7, p. 19.

24. Sagane, K., Hayakawa, K., Kai, J., Hirohashi, T. et al. (2005), 'Ataxia and peripheral nerve hypomyelination in ADAM22-deficient mice', $B M C$ Neurosi. Vol. 6, p.33.

25. Mitchell, K.J., Pinson, K.I., Kelly, O.G., Brennan, J. et al. (2001), 'Functional analysis of secreted and transmembrane proteins critical to mouse development', Nat. Genet. Vol. 28, pp. 241-249.

26. Frayne, J., Hurd, E.A. and Hall, L. (2002), 'Human tMDC III: A sperm protein with a potential role in oocyte recognition', Mol. Hum. Reprod. Vol. 8, pp. 817-822.

27. Choi, I., Woo, J.M., Hong, S., Jung, Y.K. et al. (2003), 'Identification and characterization of ADAM32 with testis-predominant gene expression', Gene Vol. 304, pp. 151-162.

28. Cong, L. and Jia, J. (2009), 'Promoter polymorphisms which regulate ADAM9 transcription are protective against sporadic Alzheimer's disease', Neurobiol. Aging in press.

29. Wang, F, Xu, R., Zhu, P., Hu, J. et al. (2001), 'Preliminarily functional analysis of a cloned novel human gene ADAM29', Sci. China C. Life Sci. Vol. 44, pp. 392-399.

30. Hooft van Huijsduijnen, R. (1998), 'ADAM 20 and 21: Two novel human testis-specific membrane metalloproteases with similarity to fertilin-alpha', Gene Vol. 206, pp. 273-282.

31. Yang, P., Baker, K.A. and Hagg, T. (2005), 'A disintegrin and metalloprotease 21 (ADAM21) is associated with neurogenesis and axonal growth in developing and adult rodent CNS', J. Comp. Neurol. Vol. 490, pp. $163-179$.

32. Cerretti, D.P., Dubose, R.F, Black, R.A. and Nelson, N. (1999), 'Isolation of two novel metalloproteinase-disintegrin (ADAM) cDNAs that show testis-specific gene expression', Biochem. Biophys. Res. Commun. Vol. 263, pp. 810-815.

33. Bates, E.E., Fridman, W.H. and Mueller, C.G. (2002), 'The $A D A M D E C 1$ (decysin) gene structure: Evolution by duplication in a metalloprotease gene cluster on chromosome 8p12', Immunogenetics Vol. 54, pp. $96-105$.

34. Zhao, Z., Tang, L., Deng, Z., Wen, L. et al. (2008), 'Essential role of ADAM28 in regulating the proliferation and differentiation of human dental papilla mesenchymal cells (hDPMCs)', Histochem. Cell Biol. Vol. 130, pp. 1015-1025.

35. Oh, J., Woo, J.M., Choi, E., Kim, T. et al. (2005), 'Molecular, biochemical, and cellular characterization of epididymal ADAMs, ADAM7 and ADAM28', 'Biochem. Biophys. Res. Commun. Vol. 331, pp. $1374-1383$

36. Mueller, C.G., Rissoan, M.C., Salinas, B., Ait-Yahia, S. et al. (1997), 'Polymerase chain reaction selects a novel disintegrin proteinase from CD40-activated germinal center dendritic cells', J. Exp. Med. Vol. 186, pp. 655-663.

37. Zhong, J.L., Poghosyan, Z., Pennington, C.J., Scott, X. et al. (2008), 'Distinct functions of natural ADAM-15 cytoplasmic domain variants in human mammary carcinoma', Mol. Cancer Res. Vol. 6, pp. 383-394.

38. Thongngarm, T., Jameekornrak, A., Limwongse, C., Sangasapaviliya, A. et al. (2008), 'Association between ADAM33 polymorphisms and asthma in a Thai population', Asian Pac. J. Allergy Immunol. Vol. 26, pp. 205-211.

39. Hirota, T., Hasegawa, K., Obara, K., Matsuda, A. et al. (2006), 'Association between ADAM33 polymorphisms and adult asthma in the Japanese population', Clin. Exp. Allergy Vol. 36, pp. 884-891. 
40. Chiba, Y., Onoda, S., Hattori, Y., Maitani, Y. et al. (2009), 'Upregulation of ADAM8 in the airways of mice with allergic bronchial asthma', Lung Vol. 187, pp. 179-185.

41. King, N.E., Zimmermann, N., Pope, S.M., Fulkerson, P.C. et al. (2004), 'Expression and regulation of a disintegrin and metalloproteinase (ADAM) 8 in experimental asthma', Am. J. Respir. Cell Mol. Biol. Vol. 31, pp. $257-265$.

42. Sahin, U., Weskamp, G., Kelly, K., Zhou, H.M. et al. (2004), 'Distinct roles for ADAM10 and ADAM17 in ectodomain shedding of six EGFR ligands', J. Cell Biol. Vol. 164, pp. 769-779.

43. Wang, Y., Herrera, A.H., Li, Y., Belani, K.K. et al. (2009), 'Regulation of mature ADAM17 by redox agents for L-selectin shedding', J. Immunol. Vol. 182, pp. 2449-2457.

44. Dierker, T., Dreier, R., Petersen, A., Bordych, C. et al. (2009), 'Heparan sulfate-modulated, metalloprotease-mediated sonic hedgehog release from producing cells', J. Biol. Chem. Vol. 284 , pp. 8013-8022.

45. Brynskov, J., Foegh, P., Pedersen, G., Ellervik, C. et al. (2002). 'Tumour necrosis factor alpha converting enzyme (TACE) activity in the colonic mucosa of patients with inflammatory bowel disease', Gut Vol. 51, pp. $37-43$.

46. Oh, S.T., Schramme, A., Stark, A., Tilgen, W. et al. (2009), 'The disintegrin-metalloproteinases ADAM 10, 12 and 17 are upregulated in invading peripheral tumor cells of basal cell carcinomas', J. Cutan. Pathol. Vol. 36, pp. 395-401.

47. Mezyk-Kopec, R., Bzowska, M., Stalinska, K., Chelmicki, T. et al. (2009), 'Identification of ADAM10 as a major TNF sheddase in ADAM17-deficient fibroblasts', Cytokine Vol. 46, pp. 309-315.

48. Porter, S., Clark, I.M., Kevorkian, L. and Edwards, D.R. (2005), 'The ADAMTS metalloproteinases', Biochem. J. Vol. 386, pp. 15-27.

49. Blelloch, R. and Kimble, J. (1999), 'Control of organ shape by a secreted metalloprotease in the nematode Caenorhabditis elegans', Nature Vol. 399, pp. $586-590$.

50. Liu, C.J. (2009), 'The role of ADAMTS-7 and ADAMTS-12 in the pathogenesis of arthritis', Nat. Clin. Pract. Rheumatol. Vol. 5, pp. $38-45$.

51. Nagase, H. and Kashiwagi, M. (2003), 'Aggrecanases and cartilage matrix degradation', Arthritis Res. Ther. Vol. 5, pp. 94-103.

52. Fosang, A.J., Rogerson, F.M., East, C.J. and Stanton, H. (2008), 'ADAMTS-5: The story so far', Eur. Cell Mater. Vol. 15, pp. 11-26.

53. Somerville, R.P., Longpre, J.M., Apel, E.D., Lewis, R.M. et al. (2004), 'ADAMTS7B, the full-length product of the ADAMTS7 gene, is a chondroitin sulfate proteoglycan containing a mucin domain', J. Biol. Chem. Vol. 279, pp. 35159-35175.
54. Nardi, J.B., Martos, R., Walden, K.K., Lampe, D.J. et al. (1999), 'Expression of lacunin, a large multidomain extracellular matrix protein, accompanies morphogenesis of epithelial monolayers in Manduca sexta, Insect Biochem. Mol. Biol. Vol. 29, pp. 883-897.

55. Kutz, W.E., Wang, L.W., Dagoneau, N., Odrcic, K.J. et al. (2008), 'Functional analysis of an ADAMTS10 signal peptide mutation in Weill-Marchesani syndrome demonstrates a long-range effect on secretion of the full-length enzyme', Hum. Mutat. Vol. 29, pp. $1425-1434$.

56. Gao, S., De Geyter, C., Kossowska, K. and Zhang, H. (2007), 'FSH stimulates the expression of the ADAMTS-16 protease in mature human ovarian follicles', Mol. Hum. Reprod. Vol. 13, pp. 465-471.

57. Li, S.W., Arita, M., Fertala, A., Bao, Y. et al. (2001), 'Transgenic mice with inactive alleles for procollagen $\mathrm{N}$-proteinase (ADAMTS-2) develop fragile skin and male sterility', Biochem. J. Vol. 355, pp. 271-278.

58. Zheng, X., Chung, D., Takayama, T.K., Majerus, E.M. et al. (2001), 'Structure of von Willebrand factor-cleaving protease (ADAMTS13), a metalloprotease involved in thrombotic thrombocytopenic purpura', J. Biol. Chem. Vol. 276, pp. 41059-41063.

59. Bork, P. and Beckmann, G. (1993), 'The CUB domain. A widespread module in developmentally regulated proteins', J. Mol. Biol. Vol. 231, pp. 539-545.

60. Hirohata, S., Wang, L.W., Miyagi, M., Yan, L. et al. (2002), 'Punctin, a novel ADAMTS-like molecule, ADAMTSL-1, in extracellular matrix', J. Biol. Chem. Vol. 277, pp. 12182-12189.

61. Le Goff, C., Morice-Picard, F, Dagoneau, N., Wang, L.W. et al. (2008), 'ADAMTSL2 mutations in geleophysic dysplasia demonstrate a role for ADAMTS-like proteins in TGF-beta bioavailability regulation', Nat. Genet. Vol. 40, pp. 1119-1123.

62. Hall, N.G., Klenotic, P., Anand-Apte, B. and Apte, S.S. (2003), 'ADAMTSL-3/punctin-2, a novel glycoprotein in extracellular matrix related to the ADAMTS family of metalloproteases', Matrix Biol. Vol. 22, pp. 501-510.

63. Koo, B.H., Hurskainen, T., Mielke, K., Aung, P.P. et al. (2007), 'ADAMTSL3/punctin-2, a gene frequently mutated in colorectal tumors, is widely expressed in normal and malignant epithelial cells, vascular endothelial cells and other cell types, and its mRNA is reduced in colon cancer', Int. J. Cancer Vol. 121, pp. 1710-1716.

64. Buchner, D.A. and Meisler, M.H. (2003), 'TSRC1, a widely expressed gene containing seven thrombospondin type I repeats', Gene Vol. 307, pp. $23-30$.

65. Ahram, D., Sato, T.S., Kohilan, A., Tayeh, M. et al. (2009), 'A homozygous mutation in ADAMTSL4 causes autosomal-recessive isolated ectopia lentis', Am. J. Hum. Genet. Vol. 84, pp. 274-278 